\title{
Rapidly progressive COVID-19 viral pneumonia: a report of two patients with a focus on imaging findings
}

\author{
Armin Zarrintan ${ }^{1,2}$, Reza Javadrashid ${ }^{1}$, Mohammad Kazem Tarzamni ${ }^{1}$, Anita Zarrintan ${ }^{2,3}$, Masih Falahatian ${ }^{2}$ and \\ Mohammad Mirza-Aghazadeh-Attari ${ }^{1,2,4^{*}}$ (D)
}

\begin{abstract}
Background: The novel coronavirus causes viral pneumonia characterized by lower respiratory tract symptoms and 19 severe inflammatory response syndrome. Studies have suggested that the virus has a clinical course with the stepwise progression of clinical signs and symptoms and radiologic alterations.

Case presentation: In the present case report, we discuss two patients who presented with mild symptoms and CT imaging not suggestive of COVID-19, but subsequently had a rapid deterioration, with severe involvement happening in CT imaging. One of the patients survived the initial deterioration, but the other passed away.

Conclusion: We suggest that the clinical course of the virus may be rapidly progressive in some patients, and special attention should be paid to patients being treated for the virus outside of the hospital as an outpatient.
\end{abstract}

Keywords: COVID-19, Novel coronavirus, Radiology, CT scan

\section{Background}

Severe acute respiratory syndrome-coronavirus-2 was the latest pandemic which started from Wuhan, China. This viral disease is characterized by pulmonary involvement, commonly manifested by coughs, rigor, and fever. Diagnosis of the disease is done by molecular assays, but CT imaging has also been proven detrimental in timely diagnosis and has shown to have an acceptable sensitivity [1]. The disease causes bilateral, multifocal groundglass opacities in the lungs. The clinical course of the disease has been studied, and it has been revealed that CT findings may be useful in determining the progression of the disease and the clinical stage of the disease. These studies are done on a limited number of patients, and most of them report information gathered from cases originating from mainland China [2].

\footnotetext{
* Correspondence: m.aghazadeh75@yahoo.com

'Department of Radiology, Tabriz University of Medical Sciences, Tabriz, Iran ${ }^{2}$ Medical Radiation Sciences Research Group, Faculty of Medicine, Tabriz University of Medical Sciences, Tabriz, Iran

Full list of author information is available at the end of the article
}

Here, we report two patients who had normal CT findings before being hospitalized but harbored symptoms suggestive of COVID-19.

\section{Case presentation}

Case one

A 63-year-old male patient presented on February 21 to the emergency department. The patient complained of fever, cough, and myalgia. The patient had diabetes mellitus from 5 years ago, with the diabetes being treated by an endocrinologist, and was otherwise healthy and had an active lifestyle. The patient reported symptoms starting from 3 days earlier. Respiratory rate was 17 , pulse rate was 93 , fever was $38{ }^{\circ} \mathrm{C}$, and blood oxygen saturation was $95 \%$ in ambient air. A chest CT scan was obtained from the patient which showed nonspecific findings (few linear and subsegmental atelectasis at lower lobes) which were not suggestive of COVID-19. The patient was put under supervision, and the molecular assay was done to determine SARS-CoV-2 infection. The results were negative, and the patient had infectious 
disease consultations done. The patient did not have the necessary criteria for hospitalization based on guidelines issued by the ministry of health, which were adapted from those of the center of disease control of the USA and world health organization ((https://irimc.org/Portals/0/NewsAttachment/\%20\%20\%20\%20\%20\%20\%20.pdf) [3, 4]). The patient presented to the emergency department on February 23 with fever and exacerbation of dyspnea and complained of symptoms, coupled with headache, nausea, and lack of appetite. The patient had a molecular assay done to rule out COVID-19, which came positive for the virus. The patient had a second CT scan, which showed no significant findings similar to the previous one. The patient was hospitalized for further evaluation regarding his fever at the internal medicine ward. On February 27, the patient had progressive dyspnea, with the continuation of fever and other mentioned symptoms. Blood oxygen saturation was measured to be 81 percent. The patient had a third CT scan, which showed bilateral diffuse ground-glass opacities and airspace consolidation suggestive for COVID-19 (Fig. 1). The patient was admitted to the intensive care unit after consultation from internists and infectious disease specialists. The patient was put on empiric antibiotic therapy and lopinavir ritonavir combined therapy. The patient had decreasing oxygen saturation levels, amide oxygen therapy using a mask with a reservoir bag. The patient was intubated on March 2 and passed away on March 3. Lab data of the patient are summarized in Table 1 .

\section{Case two}

A 52-year-old male with no pre-existing condition presented on March 21 to the emergency ward with coughs and mild myalgia from 4 days ago. The patient had a respiratory rate of 14 , a pulse rate of 76 , a body temperature of $36.7{ }^{\circ} \mathrm{C}$, and a blood pressure of $120 / 65$. The patient had contact with a COVID-19 positive case and was thus evaluated for the virus. The molecular assay revealed the patient to be infected. The patient also had a CT scan, which showed no significant finding. The patient was advised to self-quarantine in-home and did not receive any medication. The patient again presented to the emergency

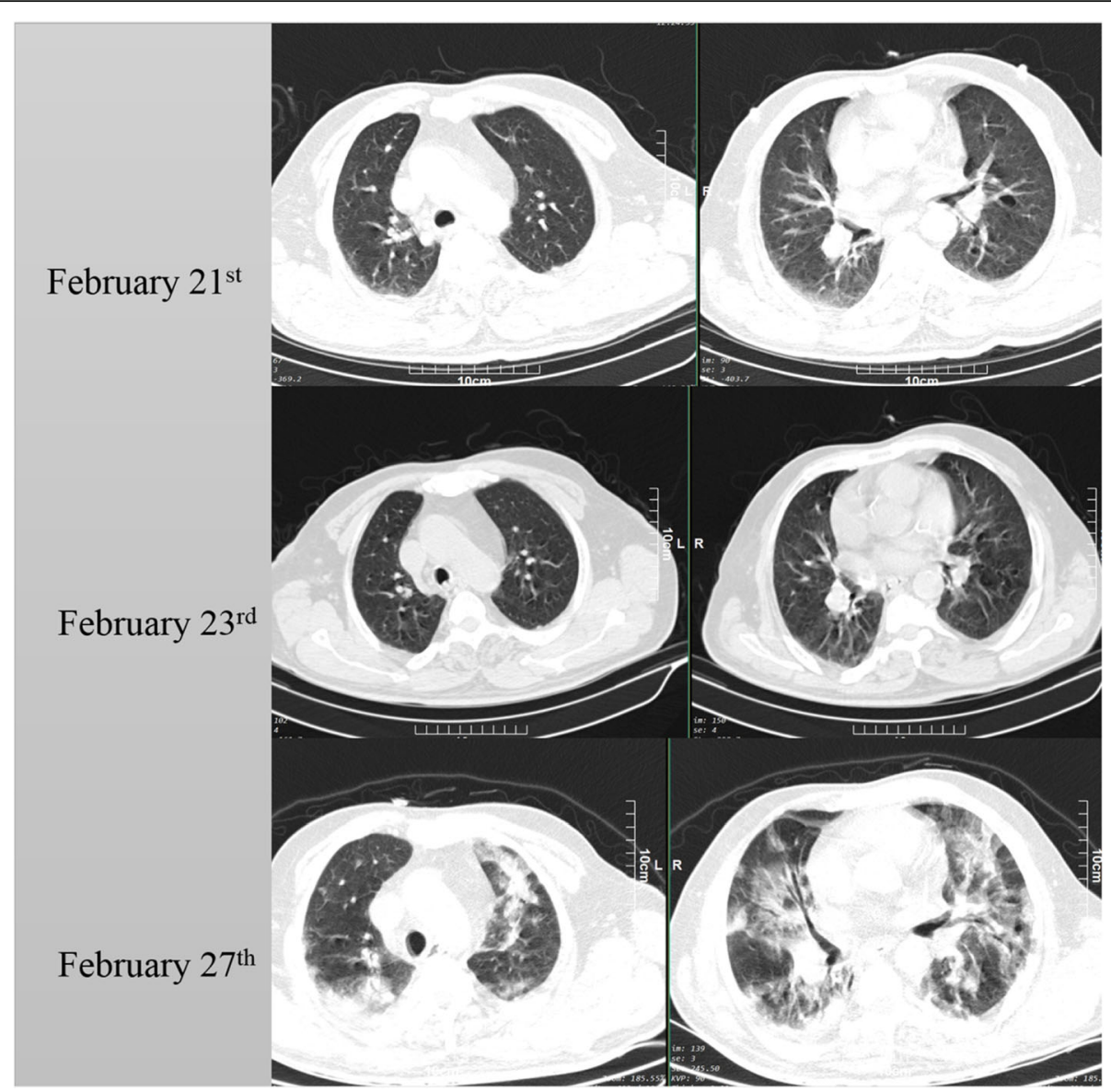

Fig. 1 The first and second rows show the chest CT scan which was obtained at the emergency department, which shows no significant findings consistent with COVID19 except some linear atelectasis. The third row shows the chest CT on February 27 which has multifocal bilateral groundglass opacities with some airspace consolidation suggestive of COVID19 
Table 1 Lab data of the patients being studied

\begin{tabular}{lllll}
\hline & Patient 1 tests on February $23^{r}$ & Patient 1 tests on February 27 & Patient 2 tests on march 26 & Patient 2 tests on march 29 \\
\hline RBC $\left(10^{6} / \mathrm{mm}^{3}\right)$ & 4.85 & 3.42 & 5.36 & 4.43 \\
$\mathrm{Hb}(\mathrm{gr} / \mathrm{dl})$ & 14.7 & 10.4 & 14.5 & 13.3 \\
Platelets $(1000 / \mathrm{mm} 3)$ & 174 & 181 & 129 & 487 \\
WBC (lymph) $\left(1000 / \mathrm{mm}^{3}\right)$ & $7.2(3.6)$ & $5.3(0.5)$ & $8.6(2.27)$ & $10.9(2.61)$ \\
Urea (mg/dl) & 50 & 95 & 25 & 23 \\
Creatinine (mg/dl) & 1.6 & 1.85 & 0.77 & 0.7 \\
AST (IU/L) & 124 & 174 & 42 & 45 \\
ALT (IU/L) & 97 & 107 & 32 & 30 \\
LDH (IU/L) & 819 & 864 & 734 & 416 \\
CPK (IU/L) & N/A & 875 & 73 & 67 \\
CRP (Quantitative) & Positive 2 & Positive 4 & Positive 3 & Positive 2 \\
ESR (mm per hour) & N/A & 121 & 104 & 87 \\
\hline
\end{tabular}

department on March 26, this time, complaining of progressive dyspnea, fever, worsening coughs, and loss of appetite. The patient had a second CT scan, which showed bilateral and diffuse multifocal ground-glass opacities and airspace consolidation with some degree of bronchiectasis in the lower lobes (Fig. 2). The patient was hospitalized in the infectious disease ward and was treated with empiric antibiotics, hydroxychloroquine, and oseltamivir. The patient was discharged from the hospital on April 1. Lab data of the patient are summarized in Table 1.

\section{Discussion}

Since the emergence of the novel coronavirus, much attention has been given to its clinical course and clinical manifestations such as the previous coronavirus pandemics [5]. Early studies have suggested that the disease has a course depending on clinical outcome and from the start of initial symptoms to discharge from hospital or death, a 19-21-day interval exists. These studies have also suggested that patients experience sepsis, ARDS, acute cardiac injury, acute kidney injury, and secondary infections before death [6]. Parallel to this, scholars have tried to suggest a radiologic staging based on disease transmission. A study conducted by Bernheim et al. classified patients into three groups based on CT imaging: Group one, those with CT imaging taken on days $0-2$ from initiation of symptoms, group two, those with images taken on days $3-5$, and the third group on days $6-12$. As the duration between CT imaging and initiation of symptoms increased, the frequency of imaging findings and severity of

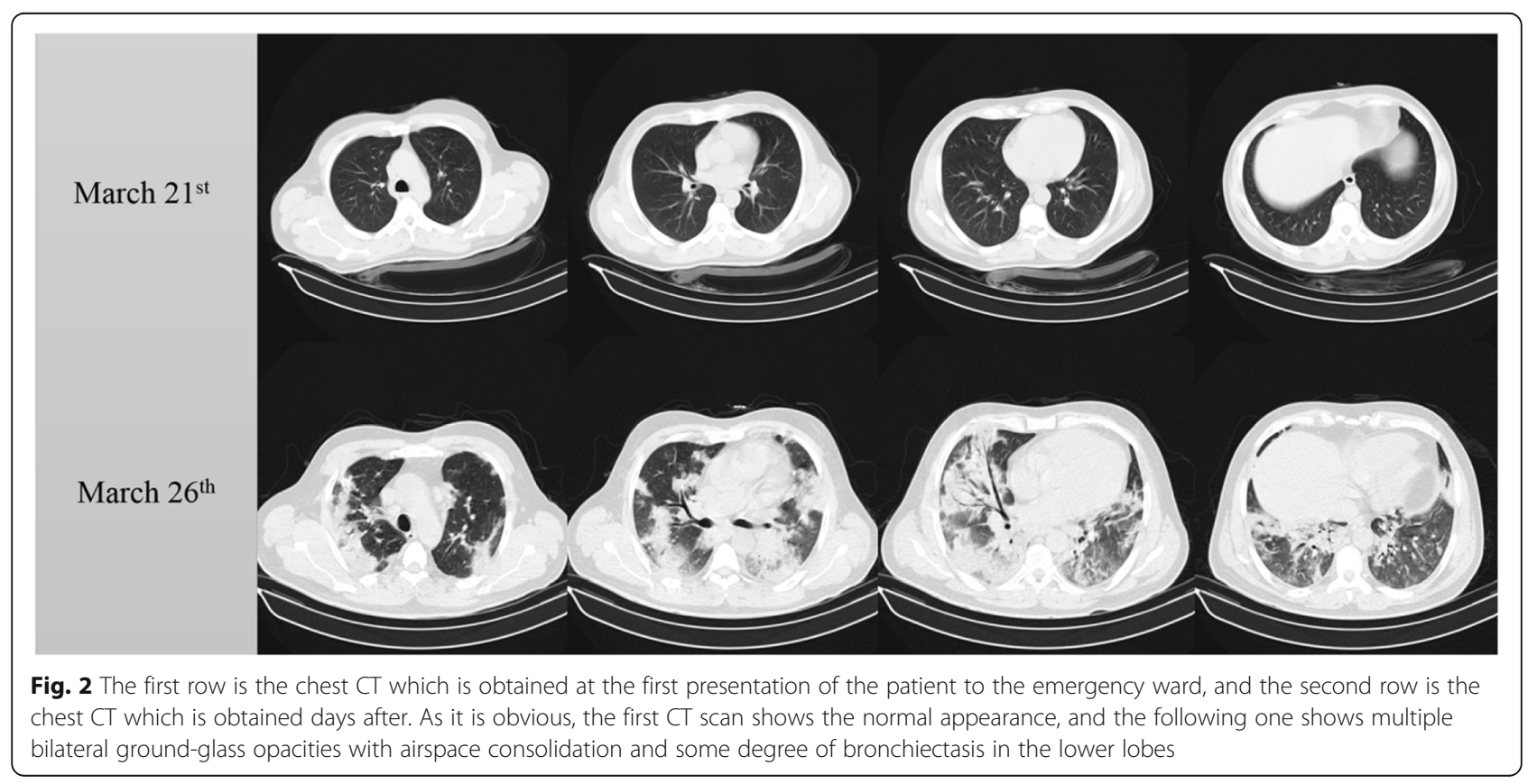


lung involvement also increased. The mean total severity score was 1,4 , and 6 for groups one, two, and three, respectively [7]. In our patients, lung involvement increased substantially in a matter of days (4 days in case one and 5 days in case two). Our findings suggest that the proposed staging can be useful in the general population, but certain patients may experience swift deterioration, both in clinical condition and radiologic findings, as our patients also had a rapid progression of clinical signs and symptoms alongside radiologic progression. Another staging system was proposed by Jin et al. This staging was based on specific radiologic findings occurring in the course of the disease. The staging consisted of 5 stages, with consolidation appearing in the second week after symptom initiation. The occurrence of consolidation was also in the second week in our patients, but early imaging findings in the first week did not occur based on the proposed timeline [8]. Furthermore, these two patients showed a rapid deterioration not previously mentioned in clinical studies [9].

\section{Conclusion}

In conclusion, the clinical course of COVID-19 may not follow that of which is presented in available scientific literature, and more attention should be paid to patients not being treated in hospitals, as they can present severe symptoms without any prior radiologic signs.

\section{Abbreviations \\ COVID-19: Coronavirus disease 2019; SARS-Cov-2: Severe acute respiratory syndrome-coronavirus-2; CT: Computed tomography; ARDS: Acute respiratory distress syndrome}

\section{Acknowledgements}

We would like to thank the radiology staff of the medical educational center in which the case underwent diagnostic imaging.

\section{Consent of publication}

Oral and written consent was taken from the patient and one of the legal representatives (in this case, a family member).

\section{Authors' contributions}

AZ: data collection, manuscript preparation, approval of the final version, and interpretation of imaging findings. RJ: data collection, obtaining the informed consent, study conception, and approval of the final version of the study. Mkt: data collection, obtaining the informed consent, study conception, and approval of the final version of the study. AnZ: obtaining the informed consent, study conception, and approval of the final version of the study. MF: data collection, obtaining the informed consent, study conception, and approval of the final version of the study. MMAA: study conception, manuscript preparation, interpretation of imaging findings, and approval of the final version of the study.

\section{Funding}

This study did not receive any funding.

\section{Availability of data and materials}

All data and materials are available based on reasonable request, according to the guidelines of the institution in which the study was approved, and based on the guidelines regarding sharing patient information.

\section{Ethics approval and consent to participate}

The study was approved by the ethical board of the medical-educational center in which it was performed.
The patient being included had signed written informed consent note. Furthermore, all patients are informed that they may be subjects of scientific experiments and their rights in this regard.

\section{Competing interests}

The authors declare no competing interests.

\section{Author details}

'Department of Radiology, Tabriz University of Medical Sciences, Tabriz, Iran. ${ }^{2}$ Medical Radiation Sciences Research Group, Faculty of Medicine, Tabriz University of Medical Sciences, Tabriz, Iran. ${ }^{3}$ Department of Internal Medicine, Faculty of Medicine, Tabriz University of Medical Sciences, Tabriz, Iran. ${ }^{4}$ Aging Research Center, Tabriz University of Medical Sciences, Tabriz, Iran.

Received: 24 April 2020 Accepted: 9 June 2020

Published online: 16 June 2020

References

1. Cascella M, Rajnik M, Cuomo A, Dulebohn SC, Di Napoli R (2020) Features, evaluation and treatment coronavirus (COVID-19). StatPearls: StatPearls Publishing

2. Huang C, Wang Y, Li X, Ren L, Zhao J, Hu Y et al (2020) Clinical features of patients infected with 2019 novel coronavirus in Wuhan, China. Lancet 395(10223):497-506

3. Murthy S, Gomersall CD, Fowler RA (2020) Care for critically ill patients with COVID-19. Jama.

4. Organization WH (2020) Critical preparedness, readiness and response actions for COVID-19: interim quidance, 22 March 2020. World Health Organization

5. Ahmadzadeh J, Mobaraki K, Mousavi SJ, Aghazadeh-Attari J, MirzaAghazadeh-Attari M, Mohebbi I (2020) The risk factors associated with MERS-CoV patient fatality: a global survey. Diagn Microbiol Infect Dis 96(3): 114876

6. Zhou F, Yu T, Du R, Fan G, Liu Y, Liu Z et al (2020) Clinical course and risk factors for mortality of adult inpatients with COVID-19 in Wuhan, China: a retrospective cohort study. Lancet

7. Bernheim A, Mei X, Huang M, Yang Y, Fayad ZA, Zhang N et al (2020) Chest CT findings in coronavirus disease-19 (COVID-19): relationship to duration of infection. Radiology. 200463

8. Jin Y-H, Cai L, Cheng Z-S, Cheng H, Deng T, Fan Y-P et al (2020) A rapid advice guideline for the diagnosis and treatment of 2019 novel coronavirus (2019-nCoV) infected pneumonia (standard version). Military Med Res 7(1):4

9. Weiss P, Murdoch DR (2020) Clinical course and mortality risk of severe COVID-19. Lancet 395(10229):1014-1015

\section{Publisher's Note}

Springer Nature remains neutral with regard to jurisdictional claims in published maps and institutional affiliations.

\section{Submit your manuscript to a SpringerOpen ${ }^{\circ}$ journal and benefit from:}

- Convenient online submission

- Rigorous peer review

- Open access: articles freely available online

- High visibility within the field

- Retaining the copyright to your article

Submit your next manuscript at $\boldsymbol{\nabla}$ springeropen.com 